\title{
Aquaglyceroporins but not orthodox aquaporins are involved in the cryotolerance of pig spermatozoa
}

\author{
Ariadna Delgado-Bermúdez, Marc Llavanera, Leira Fernández-Bastit, Sandra Recuero, Yentel Mateo-Otero, \\ Sergi Bonet, Isabel Barranco, Beatriz Fernández-Fuertes and Marc Yeste* (i)
}

\begin{abstract}
Background: Aquaporins (AQPs) are a family of transmembrane water channels that includes orthodox AQPs, aquaglyceroporins (GLPs) and superAQPs. AQP3, AQP7, AQP9 and AQP11 have been identified in boar sperm, and they are crucial for sperm maturation and osmoregulation. Water exchange is an important event in cryopreservation, which is the most efficient method for long-term storage of sperm. However, the freezethaw process leads to sperm damage and a loss of fertilizing potential. Assuming that the quality of frozenthawed sperm partially depends on the regulation of osmolality variations during this process, AQPs might play a crucial role in boar semen freezability. In this context, the aim of this study was to unravel the functional relevance of the different groups of AQPs for boar sperm cryotolerance through three different inhibitors.
\end{abstract}

Results: Inhibition of different groups of AQPs was found to have different effects on boar sperm cryotolerance. Whereas the use of 1,3-propanediol (PDO), an inhibitor of orthodox AQPs and GLPs, decreased total motility $(P<0.05)$, it increased post-thaw sperm viability, lowered membrane lipid disorder and increased mitochondrial membrane potential (MMP) $(P<0.05)$. When acetazolamide $(A C)$ was used as an inhibitor of orthodox AQPs, the effects on post-thaw sperm quality were restricted to a mild increase in MMP in the presence of the intermediate concentration at 30 min post-thaw and an increase in superoxide levels $(P<0.05)$. Finally, the addition of phloretin (PHL), a GLP inhibitor, had detrimental effects on post-thaw total and progressive sperm motilities, viability and lipid membrane disorder $(P<0.05)$.

Conclusions: The effects of the different inhibitors suggest that GLPs rather than orthodox AQPs are relevant for boar sperm freezability. Moreover, the positive effect of PDO on sperm quality suggests a cryoprotective role for this molecule.

Keywords: Acetazolamide, Aquaporins, Boar, Phloretin, Propanediol, Sperm

\section{Introduction}

Cell function and survival are strictly related to metabolite concentration, which, in turn, depends on plasma membrane permeability to water and solutes. Considering plasma membrane hydrophobicity, mechanisms other than simple diffusion are required to allow water transport across the plasma membrane in certain cell functions [1]. Aquaporins (AQPs) are a family of ubiquitous integral

\footnotetext{
* Correspondence: marc.yeste@udg.edu

Biotechnology of Animal and Human Reproduction (TechnoSperm), Unit of Cell Biology, Department of Biology, Faculty of Sciences, Institute of Food and Agricultural Technology, University of Girona, C/Maria Aurèlia Campany, 69, Campus Montilivi, E-17003 Girona, Spain
}

transmembrane proteins that allow the passive transport of water through cell membranes. Moreover, some AQPs also facilitate the transport of small solutes, such as glycerol or hydrogen peroxide [2]. Mammalian AQPs are classified according to their sequence similarity and substrate affinity into orthodox AQPs, aquaglyceroporins (GLPs) and superaquaporins (superAQPs). Orthodox $\mathrm{AQPs}$ are permeable to water, and this group is formed by AQP0, AQP1, AQP2, AQP4, AQP5, AQP6 and AQP8. Concerning the group of GLPs, it comprises AQP3, $\mathrm{AQP7}, \mathrm{AQP} 9$ and $\mathrm{AQP10}$, which are permeable to water, but also to glycerol, urea and other small electrolytes.

(c) The Author(s). 2019 Open Access This article is distributed under the terms of the Creative Commons Attribution 4.0 International License (http://creativecommons.org/licenses/by/4.0/), which permits unrestricted use, distribution, and 
Finally, AQP11 and AQP12 belong to the superAQPs group, which are specifically localized in the membrane of intracellular organelles and regulate organelle volume and intravesicular homeostasis while being involved in both water and glycerol transport [2].

AQPs have been identified in mammalian sperm cells with differences between species. While AQP3, AQP7 and AQP11 have been identified in sperm from boar $[3,4]$, mouse [5-7], human [8,9], cattle [10, 11] and stallion [12], AQP8 has only been observed in mouse [6] and human $[8,9]$ sperm, and AQP9 has been identified in boar sperm [13]. In mammalian sperm, AQPs play an important role in the adaptation to the osmotic variation caused by the exposure to the female reproductive tract, which is also involved in sperm motility activation upon ejaculation (reviewed in [2]). In addition, AQPs play a key role during spermatogenesis [14], since they contribute to cytoplasm reduction from spermatids to spermatozoa.

Cryopreservation is the most efficient method for the long-term storage of sperm. However, the freeze-thawing process damages spermatozoa in terms of plasma membrane destabilization, nuclear alterations, reduction of mitochondrial activity, changes in sperm proteins and a decrease in sperm motility $[15,16]$. Cryoinjury is mainly inflicted during the processes of freezing and thawing, when extracellular water freezes and intracellular water is lost. In this context, the penetration of permeating cryoprotective agents (CPAs), such as glycerol, is essential to minimize cryoinjuries $[15,17,18]$. The resilience of sperm cells to cryopreservation is also known as freezability or cryotolerance, which differs between species according to differences in membrane composition $[15,17,18]$. Among the AQPs identified in boar sperm cells, AQP3 and AQP7 are associated to sperm cryotolerance, which suggests that they could be used as freezability biomarkers [19].

GLPs substrate affinity and their role in volume regulation is the potential mechanism through which AQP3 and AQP7 appear to be related to sperm cryotolerance. Therefore, it is reasonable to suggest that the inhibition of sperm AQPs would impair their resilience to freezethawing procedures. Different AQP inhibitors have previously been tested in other cell types, such as 1,3-propanediol (PDO), acetazolamide (AC) and phloretin (PHL). PDO occludes the pore channel of orthodox AQPs, such as AQP1, AQP2, AQP4 and AQP5 [20, 21] from the outer side of the plasma membrane, but it is also able to penetrate through Plasmodium falciparum PfAQP (which is an analogue of AQP3, AQP7 and AQP9) and to inhibit this GLP analogue from the inner part of the plasma membrane [21]. AC has inhibitory effects on the orthodox AQPs, AQP1 and AQP4 [22, 23], whereas PHL inhibits AQP3 and AQP9, which are both GLPs [24, 25]. Against this background, this study aims to elucidate the functional relevance of orthodox AQPs and
GLPs during boar sperm cryopreservation, through their inhibition by the aforementioned agents.

\section{Methods \\ Boars and ejaculates}

A total of 20 ejaculates from separate Piétrain boars $(n=20)$ were used in this study. The boars were housed in a local farm (Semen Cardona, Cardona, Barcelona, Spain) with controlled climatic conditions, and were fed a standard diet. Sperm-rich fractions were collected manually, and subsequently diluted $1: 1(v: v)$ in a commercial semen extender (Vitasem LD; Magapor S.L., Zaragoza, Spain). After collection, ejaculates were stored in bags at $17{ }^{\circ} \mathrm{C}$ and transported to the laboratory within $5 \mathrm{~h}$ after extraction. Once in the laboratory, the ejaculates were pooled in pairs, and each pool was split into two different fractions. The first one was used to evaluate the quality parameters in fresh semen; the second one was divided into seven different sub-fractions, which were cryopreserved with or without different concentrations of the three AQP inhibitors.

\section{AQP inhibitors}

Prior to cryopreservation, three AQP inhibitors were added to the semen samples: 1,3-propanediol (PDO, Sigma-Aldrich, St. Louis, MO, USA), acetazolamide (AC, Sigma-Aldrich), and phloretin (PHL, Sigma-Aldrich). PDO was diluted in cryopreservation medium (LEYGO medium, see composition in the following section) to a working concentration of $100 \mathrm{mmol} / \mathrm{L}, \mathrm{AC}$ was diluted in dimethyl sulfoxide (DMSO, Sigma-Aldrich) to a working concentration of $450 \mathrm{mmol} / \mathrm{L}$, and PHL was diluted in methanol (Fisher Chemical, ThermoFisher Scientific; Waltham, Massachusetts, USA) to a working concentration of $365 \mathrm{mmol} / \mathrm{L}$. For each inhibitor, three different concentrations were tested: $0.1,1$ and $10 \mathrm{mmol} / \mathrm{L}$ for PDO; and 250, 500 and $1000 \mu \mathrm{mol} / \mathrm{L}$ for AC and PHL. It is worth mentioning that in the case of treatments containing PDO and AC, samples were exposed to methanol or DMSO at concentrations lower than $0.5 \%(v / v)$. These concentrations showed no detrimental effects on the sperm quality parameters (data not shown).

\section{Boar sperm cryopreservation}

Cryopreservation of boar sperm was performed to determine whether the three AQP inhibitors affected their cryotolerance. The fraction of the pool intended for cryopreservation was divided into $50-\mathrm{mL}$ aliquots and centrifuged at $15^{\circ} \mathrm{C}$ and $2400 \times g$ for $3 \mathrm{~min}$. After discarding the supernatants, pellets were resuspended to a final concentration of $1.5 \times 10^{9}$ spermatozoa $/ \mathrm{mL}$ in $\beta$-lactoseegg yolk freezing medium (LEY), which consisted of $80 \%$ $(v / v)$ lactose (Sigma-Aldrich) and 20\% $(v / v)$ egg yolk. Subsequently, sperm were cooled down to $5^{\circ} \mathrm{C}$ with a cooling 
ramp of $-0.1{ }^{\circ} \mathrm{C} / \mathrm{min}(180 \mathrm{~min})$ in a programmable, controlled-rate freezer (Icecube14S-B; Minitüb Ibérica SL; Tarragona, Spain). Sperm were then diluted to a final concentration of $1 \times 10^{9}$ spermatozoa/mL in LEYGO medium, which consisted of LEY medium supplemented with 6\% $(v / v)$ glycerol (Sigma-Aldrich) and 1.5\% Orvus ES Paste (Equex STM; Nova Chemical Sales Inc., Scituate, MA, USA). At this point, the suspension was divided into seven sub-fractions: one for each concentration and inhibitor, and a non-treated control. Samples were packed into distinct $0.5 \mathrm{~mL}$ plastic straws (Minitüb Ibérica, S.L.). Afterwards, straws were placed in a controlled-rate, programmable freezer (Icetube 14S-B; Minitub Ibérica, S.L), using the following cooling rates [26]: $-6^{\circ} \mathrm{C} / \mathrm{min}$ from 5 to $-5^{\circ} \mathrm{C}(100 \mathrm{~s}) ;-39.82^{\circ} \mathrm{C} / \mathrm{min}$ from -5 to $80^{\circ} \mathrm{C}(113 \mathrm{~s})$; hold at $-80^{\circ} \mathrm{C}$ for $30 \mathrm{~s}$; and cooled at $60^{\circ} \mathrm{C} / \mathrm{min}$ from -80 to $-150^{\circ} \mathrm{C}(70 \mathrm{~s})$. Finally, straws were plunged into liquid nitrogen $\left(-196^{\circ} \mathrm{C}\right)$ for storage.

Analysis of sperm quality parameters was performed after thawing. With this purpose, two straws per treatment and pool were immersed and agitated in a water bath at $38{ }^{\circ} \mathrm{C}$ for $15 \mathrm{~s}$. Thereafter, sperm samples were diluted 1:3 $(v: v)$ in pre-warmed Beltsville Thawing Solution (BTS) [27]. Diluted, frozen-thawed samples were incubated at $38^{\circ} \mathrm{C}$ for $240 \mathrm{~min}$, and sperm quality was evaluated at two different time points: $30 \mathrm{~min}$ and $240 \mathrm{~min}$.

\section{Sperm motility}

Sperm motility was evaluated in both fresh and frozenthawed semen samples through a computer-assisted sperm analysis (CASA) system, which consisted of a phase contrast microscope (Olympus BX41; Olympus, Tokyo, Japan) equipped with a video camera and ISAS software (Integrated Sperm Analysis System V1.0; Proiser SL, Valencia, Spain). Extended samples were incubated at $38{ }^{\circ} \mathrm{C}$ for $15 \mathrm{~min}$ before sperm motility assessment, whereas frozen-thawed samples were directly examined after $30 \mathrm{~min}$ and $240 \mathrm{~min}$ of incubation. Following incubation, $5 \mu \mathrm{L}$ of the sperm suspension were placed onto a pre-warmed Makler counting chamber (Sefi-Medical Instruments, Haifa, Israel) and observed under a negative phase-contrast field (Olympus $10 \times 0.30$ PLAN objective; Olympus). At least 1000 sperm were examined per replicate, and three replicates were evaluated per sample.

In each motility assessment, the following parameters were recorded: total (TMOT, \%) and progressive sperm motility (PMOT, \%); curvilinear velocity (VCL, $\mu \mathrm{m} / \mathrm{s})$; straight line velocity (VSL, $\mu \mathrm{m} / \mathrm{s}$ ); average path velocity (VAP, $\mu \mathrm{m} / \mathrm{s}$ ); amplitude of lateral head displacement $(\mathrm{ALH}, \mu \mathrm{m})$; beat cross frequency $(\mathrm{BCF}, \mathrm{Hz})$; linearity (LIN, \%), which was calculated assuming that $\mathrm{LIN}=\mathrm{VSL} /$ $\mathrm{VCL} \times 100$; straightness (STR, \%), resulting from VSL/
VAP $\times 100$; and motility parameter wobble (WOB, \%), obtained from VAP/VCL $\times 100$. A sperm cell was considered to be motile when its VAP was equal to or higher than $10 \mu \mathrm{m} / \mathrm{s}$ and progressively motile when its STR was equal to or higher than $45 \%$. The corresponding mean \pm standard error of the mean (SEM) was calculated for each parameter.

\section{Flow cytometry}

As mentioned above, five different parameters were assessed through flow cytometry in both fresh and frozen-thawed semen samples: viability, membrane lipid disorder, mitochondrial membrane potential (MMP), intracellular levels of superoxide $\left(\mathrm{O}_{2}^{-} \bullet\right)$ radicals and intracellular levels of hydrogen peroxide $\left(\mathrm{H}_{2} \mathrm{O}_{2}\right)$. All fluorochromes used were purchased from ThermoFisher Scientific. For proper staining, all samples were diluted to a final concentration of $1 \times 10^{6}$ spermatozoa $/ \mathrm{mL}$, and after the addition of the corresponding fluorochromes, they were incubated at $38{ }^{\circ} \mathrm{C}$ in the dark. A total of three replicates per sample were assessed for each parameter.

Samples were evaluated using a Cell Laboratory QuantaSC ${ }^{\text {tix }}$ cytometer (Beckman Coulter; Fullerton, CA, USA). All samples were excited with an argon ion laser $(488 \mathrm{~nm})$ set at a power of $22 \mathrm{~mW}$. Cell diameter/volume was assessed employing the Coulter principle for volume assessment using the Cell Lab Quanta ${ }^{\text {Tw }}$ SC cytometer, which is based on measuring changes in electrical resistance produced in an electrolyte solution by suspended, non-conductive particles. In this system, forward scatter (FS) is replaced by electronic volume (EV). Furthermore, $10-\mu \mathrm{m}$ Flow-Check fluorospheres (Beckman Coulter) were used for EV-channel calibration, by positioning this size of bead at channel 200 on the EV-scale.

Three optical filters were used: FL1 (Dichroic/Splitter, DRLP: $550 \mathrm{~nm}$, BP filter: $525 \mathrm{~nm}$, detection width: $505-$ $545 \mathrm{~nm}$ ), FL2 (DRLP: $600 \mathrm{~nm}$, BP filter: $575 \mathrm{~nm}$, detection width: $560-590 \mathrm{~nm}$ ) and FL3 (LP filter: $670 \mathrm{~nm} / 730$ $\mathrm{nm}$, detection width: $655-685 \mathrm{~nm}$ ). The first one was used to detect green fluorescence from SYBR-14, YOPRO-1, JC-1 monomers (JC-1 $1_{\text {mon }}$ ) and 2',7'-dichlorofluorescein $\left(\mathrm{DCF}^{+}\right)$; the second one was used to detect orange fluorescence from JC- 1 aggregates $\left(\mathrm{JC}-1_{\mathrm{agg}}\right)$; and the third one was used to detect red fluorescence from propidium iodide (PI), merocyanine 540 (M540) and ethidium $\left(E^{+}\right)$. The signal was logarithmically amplified, and the adjustment of photomultiplier settings was performed according to particular staining methods.

For all particles, EV and side scatter (SS) were measured and linearly recorded. The sheath flow rate was set at $4.17 \mu \mathrm{L} / \mathrm{min}$ and a minimum of 10,000 events were evaluated per replicate. On the EV channel, the analyzer threshold was adjusted to exclude subcellular debris (particle diameter $<7 \mu \mathrm{m}$ ) and cell aggregates (particle 
diameter $>12 \mu \mathrm{m})$. Therefore, on the basis of EV and SS distributions, the sperm-specific events were positively gated, whereas the others were gated out.

Subsequent data analysis was performed using Flowing Software (Ver. 2.5.1; University of Turku, Finland) and according to the recommendations of the International Society for Advancement of Cytometry (ISAC). The corresponding mean \pm SEM was calculated for each parameter.

\section{Plasma membrane integrity}

Sperm viability was evaluated by the assessment of membrane integrity using the LIVE/DEAD Sperm Viability Kit (Molecular Probes, Eugene, OR, USA), following the protocol of Garner and Johnson [28]. In brief, sperm were incubated with SYBR-14 (final concentration: $100 \mathrm{nmol} / \mathrm{L}$ ) for $10 \mathrm{~min}$, PI was subsequently added (final concentration of $12 \mu \mathrm{mol} / \mathrm{L}$ ) and sperm were incubated for an additional $5 \mathrm{~min}$. In flow cytometry dot plots, three sperm populations were observed: 1) viable, green-stained sperm $\left.\left(\mathrm{SYBR}-14^{+} / \mathrm{PI}^{-}\right) ; 2\right)$ non-viable, redstained sperm (SYBR-14- $\left./ \mathrm{PI}^{+}\right)$; 3) non-viable, both redand green-stained sperm $\left(\mathrm{SYBR}-14^{+} / \mathrm{PI}^{+}\right)$. The fourth dot population corresponded to unstained, non-sperm particles (SYBR-14- $/ \mathrm{PI}^{-}$). Viable green-stained sperm were used to assess sperm viability, and SYBR-14 fluorescence spill over into FL3-channel was compensated (2.45\%).

\section{Sperm membrane lipid disorder}

The evaluation of membrane lipid disorder was performed with M540 and YO-PRO-1 fluorochromes following a modification of the protocol from Rathi et al. [29] with minor modifications by Yeste et al. [30]. M540 detects a decrease in packing order of phospholipids in the outer monolayer of the plasma membrane. Sperm were incubated with M540 at a final concentration of $2.6 \mu \mathrm{mol} / \mathrm{L}$, and with YO-PRO- 1 at a final concentration of $25 \mathrm{nmol} / \mathrm{L}$ for $10 \mathrm{~min}$. Four populations were identified in flow cytometry dot plots: 1) non-viable sperm with low membrane lipid disorder (M540-/YO-PRO- ${ }^{+}$); 2) non-viable sperm with high membrane lipid disorder

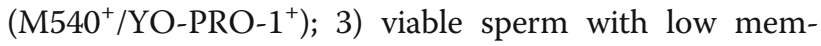
brane lipid disorder (M540- $\left./ \mathrm{YO}-\mathrm{PRO}-1^{-}\right)$; and 4) viable sperm with high membrane lipid disorder $\left(\mathrm{M} 540^{+} / \mathrm{YO}-\right.$ PRO- $1^{-}$). The percentage of viable sperm with low membrane lipid disorder (M540 ${ }^{-}$YYO-PRO- ${ }^{-}$) was corrected using the non-sperm particles from the SYBR-14/ PI co-staining. Data were not compensated.

\section{Mitochondrial membrane potential (MMP)}

Determination of mitochondrial membrane potential (MMP) was performed with $\mathrm{JC}-1$ following the procedure of OrtegaFerrusola et al. [31] with minor modifications. Sperm were incubated with $0.3 \mu \mathrm{mol} / \mathrm{L} \mathrm{JC-1}$ for $30 \mathrm{~min}$. Whereas high MMP causes JC- 1 aggregate (JC- $1_{\text {agg }}$ ) formation, in sperm cells with low MMP, JC-1 remains as a monomer $\left(\mathrm{JC}-1_{\text {mon }}\right)$. Particles showing no fluorescence (either green or orange) were gated out. Flow cytometry dot plots allowed the identification of two different sperm populations: 1) sperm with low MMP (JC-1 $\left.1_{\text {mon }}\right)$; and 2) sperm with high MMP (JC- $1_{\text {agg }}$ ). Data were compensated, as green fluorescence from FL1channel was subtracted from FL2-channel (51.70\%).

\section{Intracellular levels of superoxide $\left(\mathrm{O}_{2}^{-} \cdot\right)^{-}$}

Intracellular levels of superoxide $\left(\mathrm{O}_{2}{ }^{-} \bullet\right)$ radicals were determined with hydroethidine (HE) and YO-PRO-1 fluorochromes following a modification of the procedure from Guthrie and Welch [32]. Sperm were incubated with $4 \mu \mathrm{mol} / \mathrm{L} \mathrm{HE}$ and $40 \mathrm{nmol} / \mathrm{L}$ YO-PRO-1 for $20 \mathrm{~min}$. HE permeates the sperm plasma membrane and is oxidized by $\mathrm{O}_{2}^{-} \cdot$ to ethidium $\left(\mathrm{E}^{+}\right)$among other products. Flow cytometry dot plots allowed the identification of four different sperm populations: 1) non-viable sperm with low superoxide levels $\left(\mathrm{E}^{-} / \mathrm{YO}-\right.$ PRO- $\left.1^{+}\right)$; 2) non-viable sperm with high superoxide levels $\left(\mathrm{E}^{+} / \mathrm{YO}-\mathrm{PRO}-1^{+}\right)$; 3) viable sperm with low superoxide levels ( $\left.\mathrm{E}^{-} / \mathrm{YO}-\mathrm{PRO}-1^{-}\right)$; and 4$)$ viable sperm with high superoxide levels $\left(\mathrm{E}^{+} / \mathrm{YO}-\mathrm{PRO}-1^{-}\right)$. The percentage of viable sperm with low superoxide levels $\left(\mathrm{E}^{-} / \mathrm{YO}-\mathrm{PRO}-1^{-}\right)$was corrected using the nonsperm particles from the SYBR-14/PI co-staining. The percentages of sperm in the other three populations were recalculated and YO-PRO-1 spill over into the FL3channel was compensated (5.06\%).

\section{Intracellular levels of hydrogen peroxide $\left(\mathrm{H}_{2} \mathrm{O}_{2}\right)$}

Intracellular levels of hydrogen peroxide $\left(\mathrm{H}_{2} \mathrm{O}_{2}\right)$ were determined with $2^{\prime}, 7^{\prime}$-dichlorodihydrofluorescein diacetate $\left(\mathrm{H}_{2} \mathrm{DCFDA}\right)$ and PI fluorochromes, following the procedure from Guthrie and Welch [32] with minor modifications. Sperm were incubated with $200 \mu \mathrm{mol} / \mathrm{L}$ $\mathrm{H}_{2}$ DCFDA and $12 \mu \mathrm{mol} / \mathrm{L}$ PI for $30 \mathrm{~min}$. $\mathrm{H}_{2}$ DCFDA is a non-fluorescent probe that, after penetrating the sperm cell membrane, is intracellularly de-esterified and converted into highly fluorescent, $2^{\prime}, 7^{\prime}$-dichlorofluorescein $\left(\mathrm{DCF}^{+}\right)$upon oxidation. Flow cytometry dot plots allowed the identification of four different sperm cell populations: 1) viable sperm with high peroxide levels $\left.\left(\mathrm{DCF}^{+} / \mathrm{PI}^{-}\right) ; 2\right)$ non-viable sperm with high peroxide levels $\left(\mathrm{DCF}^{+} / \mathrm{PI}^{+}\right)$; 3) viable sperm with low peroxide levels $\left(\mathrm{DCF}^{-} / \mathrm{PI}^{-}\right)$; and 4) non-viable sperm with low peroxide levels $\left(\mathrm{DCF}^{-} / \mathrm{PI}{ }^{+}\right)$. $\mathrm{DCF}^{+}$-spill over into FL3channel was compensated (2.45\%). The percentage of viable sperm with high peroxide levels $\left(\mathrm{DCF}^{+} / \mathrm{PI}^{-}\right)$was corrected using the non-sperm particles from the SYBR14/PI co-staining. 


\section{Statistical analyses}

Data were analyzed using a statistical package (IBM SPSS Statistics 25.0; Armonk, New York, USA). Distribution of data and homogeneity of variances were tested through Shapiro-Wilk and Levene tests, respectively. A mixed model was subsequently run for each sperm parameter. The intra-subjects factor was the cryopreservation step (i.e. fresh, frozen-thawed at $30 \mathrm{~min}$, frozen-thawed at $240 \mathrm{~min}$ ), the fixed-effects factor (inter-subjects) was the treatment $(\mathrm{C}$, and different concentrations of PDO, $\mathrm{AC}$ or PHL), and the random-effects factor was the ejaculate pool. A posthoc Sidak test was used for pair-wise comparisons and the level of significance was set at $P \leq 0.05$. Data are shown as mean \pm SEM.

\section{Results}

As mentioned above, sperm quality parameters were evaluated in both fresh and frozen-thawed samples to determine the effects of the AQP inhibition during cryopreservation. Because inhibitors were added immediately before cryopreservation, no effect of treatment was observed in fresh samples in any of the variables analyzed $(P>0.05$; Figs. 1, 2, 3 and Tables 1, 2, 3).

\section{Effects of PDO on sperm quality}

Figure 1 and Table 1 show sperm quality parameters before and after freeze-thawing and the effects of PDOmediated AQP inhibition during cryopreservation.

Regardless of its concentration, PDO induced a decrease in total sperm motility $(P<0.05)$ at 240 min postthaw (Fig. 1a), but there were no differences $(P>0.05)$ between the treatments and the control in terms of progressive sperm motility at any post-thaw time point (Table 1). At 30 min post-thaw, the sperm viability (percentage of SYBR-14 $4^{+} / \mathrm{PI}^{-}$spermatozoa) of all PDO treatments was higher than in control samples $(P<0.05)$, but at $240 \mathrm{~min}$ post-thaw this difference only persisted in the $1 \mathrm{mmol} / \mathrm{L}$ treatment (Fig. 1b). Whereas the percentage of viable sperm with low membrane lipid disorder

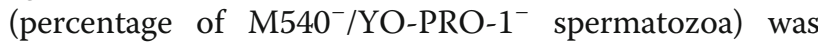
significantly $(P<0.05)$ higher than in the control at 30 min post-thaw in the presence of all PDO concentrations, only the $0.1 \mathrm{mmol} / \mathrm{L}$ and $1 \mathrm{mmol} / \mathrm{L}$ PDO concentrations elicited this effect at $240 \mathrm{~min}$ post-thaw $(P<$ 0.05; Table 1).

Moreover, addition of $0.1 \mathrm{mmol} / \mathrm{L}$ and $1 \mathrm{mmol} / \mathrm{L}$ PDO led to a higher MMP (percentage of $\mathrm{JC}^{-} 1_{\mathrm{agg}}{ }^{+}$spermatozoa) with respect to the other treatments and the control at both time points after thawing $(P<0.05$; Table 1$)$.
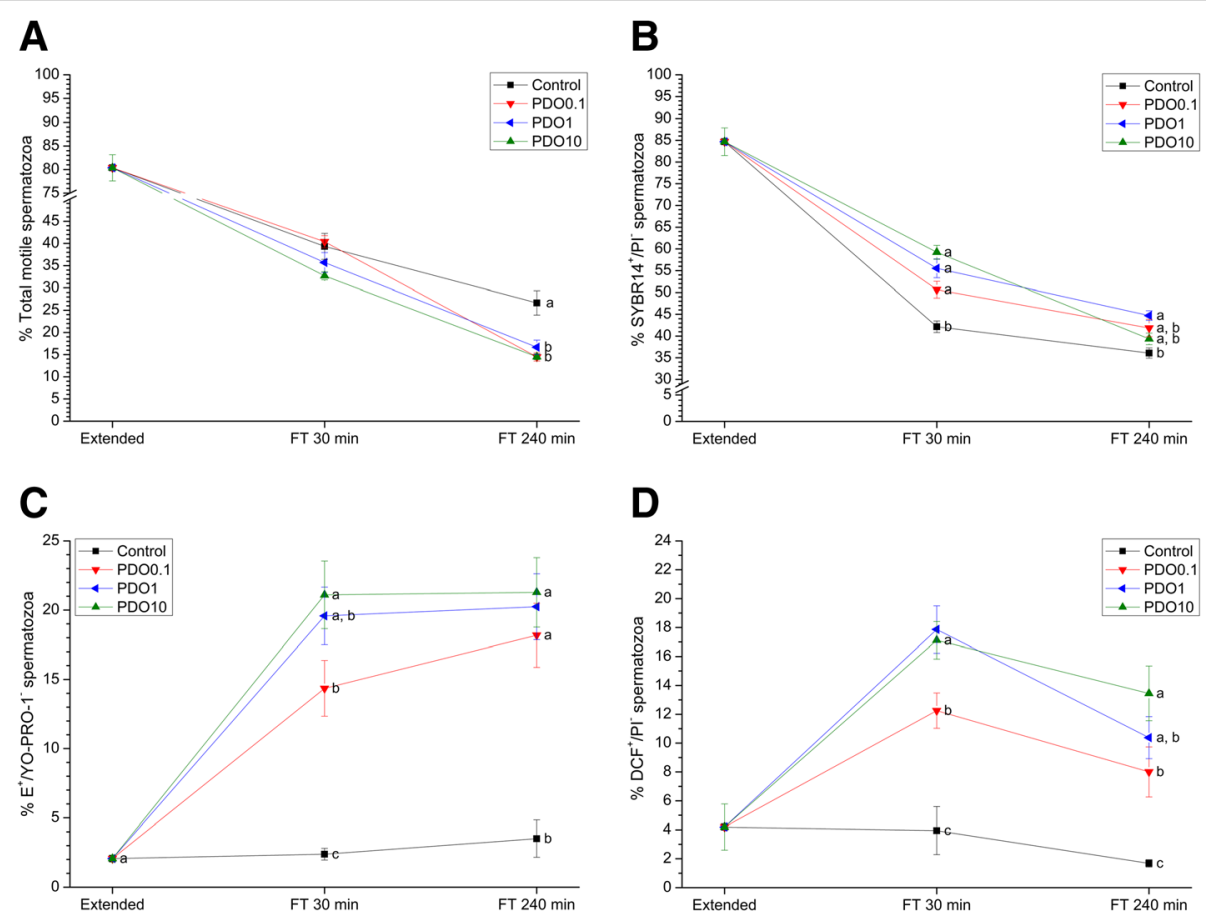

Fig. 1 Sperm quality parameters in the presence of 1,3-propanediol (PDO) at three different concentrations $(0.1 \mathrm{mmol} / \mathrm{L}, 1 \mathrm{mmol} / \mathrm{L}$ and $10 \mathrm{mmol} /$ L) compared to samples exposed to extender alone. a Percentage of total motile spermatozoa (TMOT); $\mathbf{b}$ Percentage of viable sperm (SYBR-14 ${ }^{+} /$ $\mathrm{PI}^{-}$); $\mathbf{c}$ Superoxide levels (percentage of spermatozoa E $\left./ \mathrm{YO}-\mathrm{PRO}-1^{-}\right)$; $\mathbf{d}$ Peroxide levels (percentage of spermatozoa DCF $/ \mathrm{PI}^{-}$). Data were collected from fresh (extended) and frozen-thawed (FT) samples 30 and $240 \mathrm{~min}$ after thawing. Data reported as mean \pm SEM. Different letters $(\mathbf{a}-\mathbf{c})$ indicate significant differences $(P<0.05)$ between treatments within a given time point 

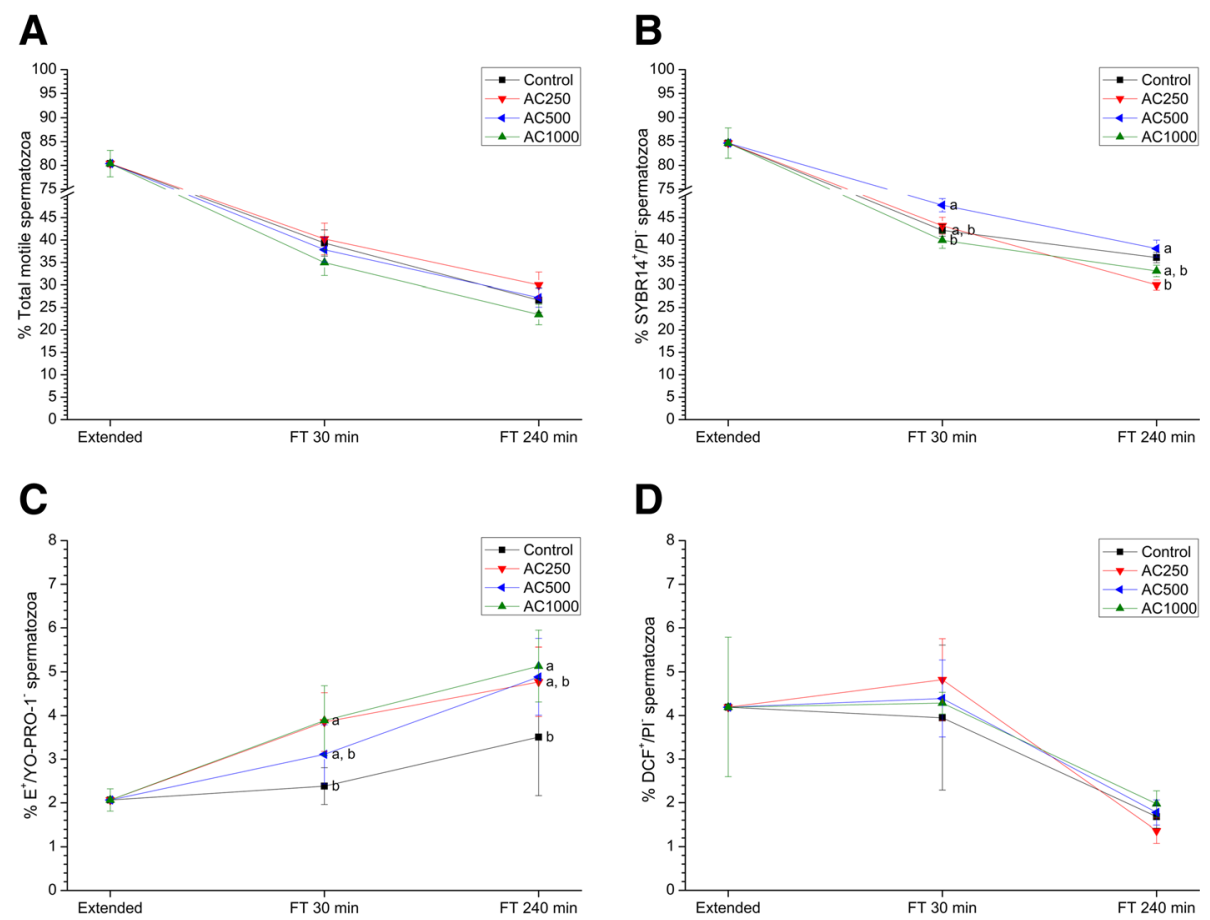

Fig. 2 Sperm quality parameters in the presence of acetazolamide (AC) at three different concentrations $(250 \mu \mathrm{mol} / \mathrm{L}, 500 \mu \mathrm{mol} / \mathrm{L}$ and $1000 \mu \mathrm{mol} /$ L) compared to samples exposed to extender alone. a Percentage of total motile spermatozoa (TMOT); b Percentage of viable sperm (SYBR-14 ${ }^{+} /$ $\mathrm{PI}^{-}$); c Superoxide levels (percentage of spermatozoa E ${ }^{+} / \mathrm{YO}-\mathrm{PRO}-1^{-}$); $\mathbf{d}$ Peroxide levels (percentage of spermatozoa $\mathrm{DCF}^{+} / \mathrm{PI}^{-}$). Data were collected from fresh (extended) and frozen-thawed ( $F T$ ) samples 30 and 240 min after thawing. Data reported as mean \pm SEM. Different letters (a, b) indicate significant differences $(P<0.05)$ between treatments within a given time point

The percentage of viable spermatozoa showing high levels of $\mathrm{O}_{2}{ }^{-}$. (percentage of $\mathrm{E}^{+} / \mathrm{YO}-\mathrm{PRO}-1^{-}$spermatozoa) was higher in PDO-supplemented samples than the control, at both 30 and 240 min post-thaw $(P<0.05$; Fig. 1c). Similarly, samples treated with different concentrations of PDO presented a higher percentage of viable sperm cells with high peroxide levels (percentage of $\mathrm{DCF}^{+} / \mathrm{PI}^{-}$spermatozoa) than the control at both 30 and 240 min post-thaw $(P<0.05$; Fig. $1 d)$.

\section{Effects of $A C$ on sperm quality}

Figure 2 and Table 2 show sperm quality parameters before and after freeze-thawing and the effects of AC-mediated AQP inhibition during cryopreservation.

Neither total nor progressive sperm motility differed between treatments containing AC and the control $(P>$ 0.05) (Fig. 2a; Table 2). Regarding viability, it decreased when sperm were exposed to $250 \mu \mathrm{mol} / \mathrm{L} \mathrm{AC}$ at $240 \mathrm{~min}$ post-thaw $(P<0.05$; Fig. $2 \mathrm{~b})$. Treatment of sperm with AC did not induce changes $(P>0.05)$ in membrane lipid disorder in any of the studied time points compared to the control (Table 2).

In the treatment containing $500 \mu \mathrm{mol} / \mathrm{L} \mathrm{AC}$, the percentage of sperm cells with high MMP was higher than in the control at 30 min post-thaw $(P<0.05$; Table 2$)$.
In addition, 250 and $1000 \mu \mathrm{mol} / \mathrm{L} \mathrm{AC}$ induced an increase in the percentage of viable sperm cells with high $\mathrm{O}_{2}{ }^{-}$- levels at $30 \mathrm{~min}$ post-thaw $(P<0.05)$, but this difference only persisted for the $1000 \mu \mathrm{mol} / \mathrm{L}$ at $240 \mathrm{~min}$ post-thaw $(P<0.05$; Fig. $2 \mathrm{c})$. In contrast, $A C$ had no effect on the percentage of viable spermatozoa with high peroxide levels (Fig. 2d).

\section{Effects of PHL on sperm quality}

Figure 3 and Table 3 show sperm quality parameters before and after freeze-thawing and the effects of PHL-mediated AQP inhibition during cryopreservation.

Concerning $\mathrm{PHL}, 500 \mu \mathrm{mol} / \mathrm{L}$ concentration reduced total sperm motility at $240 \mathrm{~min}$ post-thaw (Fig. 3a; $P<$ $0.05)$. Moreover, PHL at $1000 \mu \mathrm{mol} / \mathrm{L}$ caused a significant $(P<0.05)$ decrease in both total and progressive sperm motilities at any time point after thaw (Fig. 3a, Table 3). Similarly, $1000 \mu \mathrm{mol} / \mathrm{L}$ PHL lowered both sperm viability at $240 \mathrm{~min}$ post-thaw $(P<0.05$; Fig. $3 \mathrm{~b})$ and the percentage of viable spermatozoa with low membrane lipid disorder at any time point after thaw $(P<0.05$; Table 3$)$.

With regard to $M M P$, no significant differences $(P>$ $0.05)$ were observed in this sperm parameter between treatments and the control (Table 3).

Finally, PHL had no effect either on the percentage of viable spermatozoa with high superoxide levels or on the 
A

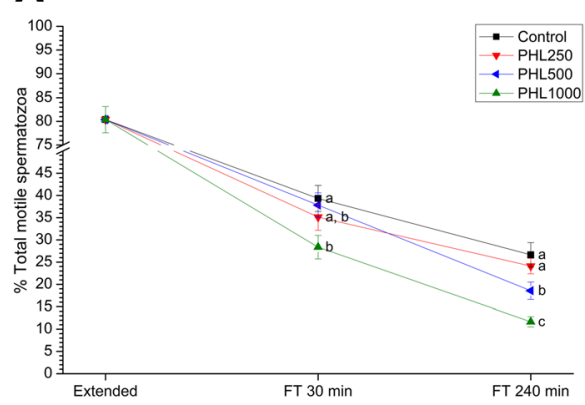

C

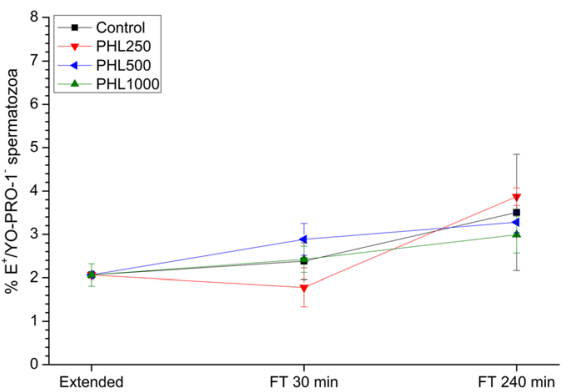

B

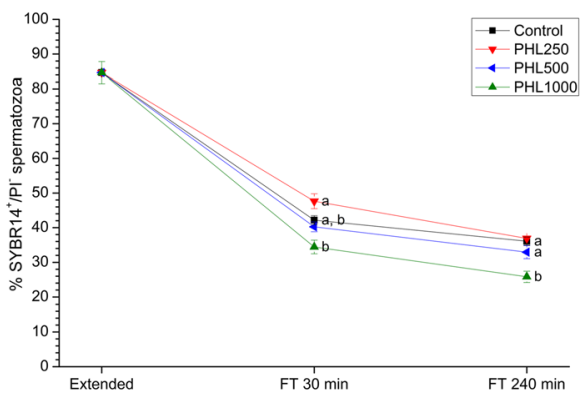

D

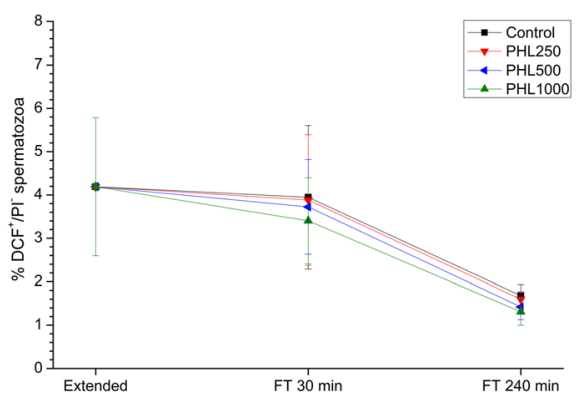

Fig. 3 Sperm quality parameters in the presence of phloretin (PHL) at three different concentrations $(250 \mu \mathrm{mol} / \mathrm{L}, 500 \mu \mathrm{mol} / \mathrm{L}$ and $1000 \mu \mathrm{mol} /$ L) compared to samples exposed to extender alone. a Percentage of total motile spermatozoa (TMOT); $\mathbf{b}$ Percentage of viable sperm (SYBR-14 $\%$ $\mathrm{PI}^{-}$); c Superoxide levels (percentage of spermatozoa $E^{+} / \mathrm{YO}^{-\mathrm{PRO}-1^{-}}$); $\mathbf{d}$ Peroxide levels (percentage of spermatozoa DCF $\mathrm{PII}^{-}$). Data were collected from fresh (extended) and frozen-thawed (FT) samples 30 and 240 min after thawing. Data reported as mean \pm SEM. Different letters (a-c) indicate significant differences $(P<0.05)$ between treatments within a given time point

Table 1 Sperm quality parameters from samples exposed to extender alone (control), or in the presence of 1,3-propanediol (PDO) at three different concentrations $(0.1 \mathrm{mmol} / \mathrm{L}, 1 \mathrm{mmol} / \mathrm{L}$ and $10 \mathrm{mmol} / \mathrm{L})$. The evaluated parameters were: percentage of progressively motile spermatozoa (PMOT), percentage of spermatozoa with low membrane lipid disorder (\% $\% 540^{-} / \mathrm{YO}^{-\mathrm{PRO}-1^{-}}$ sperm cells) and percentage of spermatozoa with high mitochondrial membrane potential (MMP; \% $\mathrm{C}_{\text {agg }}$ spermatozoa). Determinations were performed in fresh (extended) and frozen-thawed (FT) spermatozoa after 30 and 240 min of thawing. Data reported as mean \pm SEM

\begin{tabular}{|c|c|c|c|c|}
\hline \multirow[t]{2}{*}{ Variable } & \multirow{2}{*}{$\begin{array}{l}\text { PDO } \\
\text { concentration }\end{array}$} & \multicolumn{3}{|l|}{ Time point } \\
\hline & & Extended & FT-30 min & FT-240 min \\
\hline \multirow[t]{4}{*}{ \%РMOT } & CNT & $69.87 \pm 3.20$ & $23.38 \pm 2.74^{\mathrm{a}}$ & $10.96 \pm 1.12^{\mathrm{a}, \mathrm{b}}$ \\
\hline & $0.1 \mathrm{mmol} / \mathrm{L}$ & & $26.62 \pm 2.10^{a}$ & $7.60 \pm 0.42^{b}$ \\
\hline & $1 \mathrm{mmol} / \mathrm{L}$ & & $22.80 \pm 2.12^{\mathrm{a}}$ & $11.71 \pm 1.07^{\mathrm{a}}$ \\
\hline & $10 \mathrm{mmol} / \mathrm{L}$ & & $24.94 \pm 2.20^{a}$ & $8.48 \pm 0.45^{a, b}$ \\
\hline \multirow[t]{4}{*}{$\% \mathrm{M} 540^{-} / \mathrm{YO}^{\mathrm{PRO}} \mathbf{1}^{-}$} & CNT & $80.83 \pm 2.79$ & $39.03 \pm 1.49^{\mathrm{a}}$ & $31.25 \pm 1.06^{a}$ \\
\hline & $0.1 \mathrm{mmol} / \mathrm{L}$ & & $48.12 \pm 1.75^{b}$ & $37.56 \pm 1.50^{b, c}$ \\
\hline & $1 \mathrm{mmol} / \mathrm{L}$ & & $53.28 \pm 2.10^{b}$ & $42.93 \pm 1.38^{c}$ \\
\hline & $10 \mathrm{mmol} / \mathrm{L}$ & & $56.02 \pm 2.32^{b}$ & $36.50 \pm 1.23^{a, b}$ \\
\hline \multirow[t]{4}{*}{$\% \mathrm{JCl}_{\text {agg }}$} & CNT & $82.79 \pm 2.41$ & $31.95 \pm 1.89^{a}$ & $26.01 \pm 1.44^{\mathrm{a}}$ \\
\hline & $0.1 \mathrm{mmol} / \mathrm{L}$ & & $48.76 \pm 1.85^{b}$ & $38.67 \pm 1.58^{b}$ \\
\hline & $1 \mathrm{mmol} / \mathrm{L}$ & & $40.95 \pm 2.07^{c}$ & $34.39 \pm 1.54^{b, c}$ \\
\hline & $10 \mathrm{mmol} / \mathrm{L}$ & & $35.89 \pm 2.06^{a, c}$ & $30.56 \pm 1.29^{a, c}$ \\
\hline
\end{tabular}


Table 2 Sperm quality parameters from samples exposed to extender alone (control), or in the presence of acetazolamide (AC) at three different concentrations $(250 \mu \mathrm{mol} / \mathrm{L}, 500 \mu \mathrm{mol} / \mathrm{L}$ and $1000 \mu \mathrm{mol} / \mathrm{L})$. The evaluated parameters were: percentage of progressively motile spermatozoa (PMOT), percentage of spermatozoa with low membrane lipid disorder (\% $\% 540^{-} \mathrm{MO}^{-\mathrm{PRO}} \mathrm{1}^{-}$ sperm cells) and percentage of spermatozoa with high mitochondrial membrane potential (MMP; \%JC1 ${ }_{\text {agg }}$ spermatozoa). Determinations were performed in fresh (extended) and frozen-thawed (FT) spermatozoa after 30 and 240 min of thawing. Data reported as mean \pm SEM

\begin{tabular}{|c|c|c|c|c|}
\hline \multirow[t]{2}{*}{ Variable } & \multirow{2}{*}{$\begin{array}{l}\mathrm{AC} \\
\text { concentration }\end{array}$} & \multicolumn{3}{|l|}{ Time point } \\
\hline & & Extended & FT-30 min & FT-240 min \\
\hline \multirow[t]{4}{*}{ \%РMOT } & CNT & $69.87 \pm 3.20$ & $23.38 \pm 2.74^{a}$ & $10.96 \pm 1.12^{\mathrm{a}, \mathrm{b}}$ \\
\hline & $250 \mu \mathrm{mol} / \mathrm{L}$ & & $25.34 \pm 2.83^{\mathrm{a}}$ & $13.47 \pm 2.19^{\mathrm{a}}$ \\
\hline & $500 \mu \mathrm{mol} / \mathrm{L}$ & & $22.99 \pm 2.57^{a}$ & $12.94 \pm 1.98^{\mathrm{a}, \mathrm{b}}$ \\
\hline & $1000 \mu \mathrm{mol} / \mathrm{L}$ & & $20.77 \pm 2.29^{a}$ & $8.93 \pm 1.30^{b}$ \\
\hline \multirow[t]{4}{*}{ 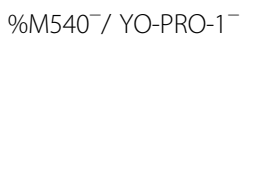 } & CNT & $80.83 \pm 2.79$ & $39.03 \pm 1.49^{a, b}$ & $31.25 \pm 1.06^{a, b}$ \\
\hline & $250 \mu \mathrm{mol} / \mathrm{L}$ & & $40.26 \pm 1.73^{\mathrm{a}, \mathrm{b}}$ & $27.01 \pm 0.98^{\mathrm{a}}$ \\
\hline & $500 \mu \mathrm{mol} / \mathrm{L}$ & & $46.39 \pm 1.30^{a}$ & $35.28 \pm 1.71^{b}$ \\
\hline & $1000 \mu \mathrm{mol} / \mathrm{L}$ & & $36.03 \pm 1.58^{b}$ & $30.52 \pm 1.18^{a, b}$ \\
\hline \multirow[t]{4}{*}{$\% J C 1_{\text {agg }}$} & CNT & $82.79 \pm 2.41$ & $31.95 \pm 1.89^{\mathrm{a}}$ & $26.01 \pm 1.44^{\mathrm{a}, \mathrm{b}}$ \\
\hline & $250 \mu \mathrm{mol} / \mathrm{L}$ & & $29.30 \pm 1.63^{\mathrm{a}}$ & $23.70 \pm 1.24^{a, b}$ \\
\hline & $500 \mu \mathrm{mol} / \mathrm{L}$ & & $40.84 \pm 2.11^{b}$ & $31.98 \pm 1.89^{a}$ \\
\hline & $1000 \mu \mathrm{mol} / \mathrm{L}$ & & $30.20 \pm 1.72^{\mathrm{a}}$ & $21.92 \pm 1.35^{b}$ \\
\hline
\end{tabular}

Different letters $\left({ }^{\mathrm{a}, \mathrm{b}}\right)$ indicate significant differences $(P<0.05)$ between treatments within a given time point for each parameter

percentage of viable spermatozoa with high peroxide levels $(P>0.05$; Fig. 3c, d).

\section{Discussion}

Despite studies conducted in the last decade identifying the presence of AQPs in sperm cells of several mammalian species (reviewed in [17]), neither their precise function nor their mechanism of action have been fully addressed. To this end, this study has used different AQP inhibitors to unveil the relevance of orthodox AQPs and GLPs during cryopreservation. Three different inhibitors were added at three concentrations each: 1,3-propanediol (PDO; 0.1, 1 and $10 \mathrm{mmol} / \mathrm{L}$ ), acetazolamide (AC; 250, 500 and $1000 \mu \mathrm{mol} / \mathrm{L})$ and phloretin

Table 3 Sperm quality parameters from samples exposed to extender alone (control), or in the presence of phloretin (PHL) at three different concentrations $(250 \mu \mathrm{mol} / \mathrm{L}, 500 \mu \mathrm{mol} / \mathrm{L}$ and $1000 \mu \mathrm{mol} / \mathrm{L})$. The evaluated parameters were: percentage of progressively motile spermatozoa (PMOT), percentage of spermatozoa with low membrane lipid disorder (\% $\% 540^{-} / \mathrm{YO}^{-\mathrm{PRO}-1^{-}}$sperm cells) and percentage of spermatozoa with high mitochondrial membrane potential (MMP; \%JC1 agg spermatozoa). Determinations were performed in fresh (extended) and frozen-thawed (FT) spermatozoa after 30 and 240 min of thawing. Data reported as mean \pm SEM

\begin{tabular}{|c|c|c|c|c|}
\hline \multirow[t]{2}{*}{ Variable } & \multirow{2}{*}{$\begin{array}{l}\mathrm{PHL} \\
\text { concentration }\end{array}$} & \multicolumn{3}{|l|}{ Time point } \\
\hline & & Extended & FT-30 min & FT-240 min \\
\hline \multirow[t]{4}{*}{$\%$ \%МOT } & CNT & $69.87 \pm 3.20$ & $23.38 \pm 2.74^{a}$ & $10.96 \pm 1.12^{\mathrm{a}}$ \\
\hline & $250 \mu \mathrm{mol} / \mathrm{L}$ & & $20.52 \pm 0.98^{\mathrm{a}}$ & $8.61 \pm 0.93^{\mathrm{a}}$ \\
\hline & $500 \mu \mathrm{mol} / \mathrm{L}$ & & $22.16 \pm 0.75^{a}$ & $7.62 \pm 0.89^{a}$ \\
\hline & $1000 \mu \mathrm{mol} / \mathrm{L}$ & & $10.38 \pm 0.90^{b}$ & $3.82 \pm 0.92^{b}$ \\
\hline \multirow[t]{4}{*}{$\%{\mathrm{M} 540^{-} / \mathrm{YO}^{-P R O}-1^{-}}^{-}$} & CNT & $80.83 \pm 2.79$ & $39.03 \pm 1.49^{a, b}$ & $31.25 \pm 1.06^{\mathrm{a}}$ \\
\hline & $250 \mu \mathrm{mol} / \mathrm{L}$ & & $44.53 \pm 1.99^{\mathrm{a}}$ & $34.19 \pm 1.29^{\mathrm{a}}$ \\
\hline & $500 \mu \mathrm{mol} / \mathrm{L}$ & & $37.92 \pm 1.37^{\mathrm{b}}$ & $30.20 \pm 1.10^{a}$ \\
\hline & $1000 \mu \mathrm{mol} / \mathrm{L}$ & & $31.01 \pm 1.60^{c}$ & $21.42 \pm 1.05^{b}$ \\
\hline \multirow[t]{4}{*}{$\% \mathrm{Cl}_{\text {agg }}$} & CNT & $82.79 \pm 2.41$ & $31.95 \pm 1.89^{\mathrm{a}}$ & $26.01 \pm 1.44^{\mathrm{a}}$ \\
\hline & $250 \mu \mathrm{mol} / \mathrm{L}$ & & $38.21 \pm 2.08^{a}$ & $22.49 \pm 1.21^{\mathrm{a}}$ \\
\hline & $500 \mu \mathrm{mol} / \mathrm{L}$ & & $34.98 \pm 2.09^{a}$ & $29.29 \pm 1.74^{a}$ \\
\hline & $1000 \mu \mathrm{mol} / \mathrm{L}$ & & $31.93 \pm 1.88^{\mathrm{a}}$ & $25.18 \pm 1.29^{\mathrm{a}}$ \\
\hline
\end{tabular}


(PHL; 250, 500 and $1000 \mu \mathrm{mol} / \mathrm{L}$ ). PDO has been proven to inhibit orthodox AQPs (AQP1, AQP2, AQP5 and AQP4) with high efficiency, and GLPs (the family to which AQP3, AQP7 and AQP9 belong) with low intensity $[20,21]$. With regard to $A C$, it inhibits $A Q P 1$ and AQP4 [22, 23], whereas PHL inhibits both AQP3 and AQP7 [33-35]. The concentrations were based on preliminary experiments conducted to determine the minimum concentration at which an effect was observed, and the maximum concentration at which cytotoxic effects appeared. The assessment of how each inhibitor affected sperm function and survival during cryopreservation was made on the basis of sperm motility, sperm viability, membrane lipid disorder, MMP, and intracellular levels of superoxide and peroxides.

Compared to the control, PDO tended to maintain better post-thaw sperm viability and lower levels of membrane lipid disorder; however, total sperm motility decreased, whereas percentages of spermatozoa with high MMP and with high levels of ROS (including superoxides and peroxides) increased at some concentrations. As far as AC is concerned, there was a lack of consistent effects, and only the percentage of spermatozoa with high MMP and intracellular levels of $\mathrm{O}_{2}{ }^{-} \cdot$ changed at some concentrations and time points. Finally, PHL had detrimental effects on total and progressive sperm motilities, viability and membrane lipid disorder.

The different effects observed between inhibitors might be due to their specificity for different AQPs and the collateral effects on other proteins that are present in the sperm cell. In this context, PDO inhibits a great number of AQPs, especially orthodox AQPs, and, with less efficiency, GLPs. Whereas PDO remains inside the pore of orthodox AQPs because of its narrow diameter, it is able to freely permeate through the GLP pore $[20,21]$. Relate to this, it is worth mentioning that Cooper et al. [36] assessed the ability of PDO to penetrate epididymal murine sperm. PDO may disrupt the transport of water and also that of small solutes, including glycerol. Since glycerol is present in many cryopreservation extenders, including the one used in this study, it is possible that its entry to sperm cells and, as a consequence, its function as a CPA, are partially impaired through GLP inhibition. Nevertheless, this hypothesis would not explain why post-thaw sperm viability and the percentage of spermatozoa with low membrane lipid disorder were higher in those samples containing PDO. In this context, one could suggest that PDO could mitigate the negative effects of the lower intracellular concentration of glycerol inside the sperm cell by acting as a CPA itself. In addition, it is widely known that glycerol, much like other CPAs, presents certain toxic effects for sperm cells (reviewed in [15]), and reducing its intracellular concentration could decrease its toxicity. Considering the aforementioned, our results could be explained by PDO lowering the toxic effect of glycerol, and acting as a CPA itself. Consequently, PDO should be further studied as a potential CPA in combination with other agents, as its positive effects on frozen-thawed sperm warrant further research and its impact on sperm viability observed in the current work is coincident with that observed by Widiasih et al. [37] in human sperm. Moreover, PDO has also been used in cryopreservation protocols for canine ovarian cortices [38] and in human multipotent stromal cells [39].

On the other hand, the increase of MMP and ROS levels in PDO-treatments is noteworthy. To the best of our knowledge, the potential effects of PDO on oxidative stress have not been previously reported. Our results suggest that the increase in sperm viability observed when this inhibitor is present in the cryopreservation medium is at the expense of an increase in the percentage of sperm cells with high levels of ROS, and therefore, with potential DNA damage [40]. It is worth mentioning that some studies have unraveled the fact that AQP3 and AQP9 are permeable to $\mathrm{H}_{2} \mathrm{O}_{2}$ [41, 42], and that $\mathrm{H}_{2} \mathrm{O}_{2}$ transport through these proteins does play a vital role in human sperm function [9]. Since the increase of MMP causes an increase in ROS production, including $\mathrm{H}_{2} \mathrm{O}_{2}$, one could suggest that the inhibition of AQP3 and AQP9 by PDO would block $\mathrm{H}_{2} \mathrm{O}_{2}$-efflux. That being said, the aforementioned increase in MMP does not have an apparent cause. A possible explanation could be an interaction of PDO with AQP11, which localizes in the mitochondrial membrane of boar sperm [3]. However, since the inhibiting capacity of PDO with regard to superAQPs has not been previously tested, further studies are needed to unravel the mechanism behind the PDO-mediated increase in MMP.

Regarding the inhibitory mechanism of PHL, it permeates the sperm plasma membrane thanks to its hydrophobic nature [43], and it inhibits GLPs through an internal binding site [44]. Considering its proven inhibitory effect on GLPs, detrimental effects on sperm quality in its presence could be a consequence of the decrease in glycerol permeability, which is consistent with the effects observed in the presence of PDO. In fact, total sperm motility is similarly affected by both inhibitors. Moreover, the detrimental effects on sperm viability and membrane lipid disorder support the hypothesis that PDO rather than PHL exerts a cryoprotective effect. Nevertheless, it is worth highlighting that PHL has inhibitory effects on other sperm proteins, such as SLC2A2 (also known as GLUT2), protein kinase C (PKC), and volume-sensitive and cAMP-activated $\mathrm{Cl}^{-}$ channels [45]. All of these proteins can play an 
important role in the regulation of sperm function: SLC2A2 is involved in the uptake of monosaccharides, which are the main energy source for sperm cells [46]; PKC is implicated in the regulation of sperm motility through phosphorylation of other sperm proteins; and chloride-dependent transport mechanisms are relevant for different sperm cell pathways, such as cAMP-protein kinase A (PKA), which plays a vital role during sperm capacitation [16]. Therefore, not only could PHL mediate the decrease in sperm motility, viability and MMP through the inhibition of GLPs, but also through the inhibition of other sperm proteins.

Concerning AC and its specificity for orthodox AQPs, one would expect that this inhibitor would not affect CPA transport, which could explain why sperm viability and membrane lipid disorder were not affected. Moreover, the fact that AC inhibits AQP1 and AQP4, which have not been previously identified in boar spermatozoa, supports the absence of effects when this inhibitor is added before cryopreservation. In spite of this, some sperm parameters were altered in the presence of AC. While this impact did not appear to depend on the AC concentration, we suggest that it is not directly related to AQP inhibition. Indeed, AC is known to inhibit carbonic anhydrase (CA), and therefore it blocks the conversion of $\mathrm{CO}_{2}$ and $\mathrm{H}_{2} \mathrm{O}$ into bicarbonate and protons [47]. Since bicarbonate together with $\mathrm{Ca}^{2+}$ stimulates PKA, and complex IV of the electron transport chain is activated via a PKA-mediated phosphorylation, inhibition of $\mathrm{CA}$ through $\mathrm{AC}$ could cause uncoupling of the electron transport chain [48]. This uncoupling is likely to be responsible for the increase in percentages of spermatozoa with high MMP and with high superoxide levels in AC treatments. Nevertheless, while the intermediate concentration of $\mathrm{AC}$ causes an increase in MMP, the highest one has no effect in this parameter compared to the control. Even if no significant differences were found in sperm viability when AC treatments and the control were compared, samples treated with the intermediate concentration of AC showed a significantly $(P<0.05)$ higher viability than those treated with the highest concentration of AC at $30 \mathrm{~min}$ post-thaw. Therefore, the absence of differences in MMP in the presence of the highest concentration of $\mathrm{AC}$ could be a consequence of mitochondrial alterations that would compromise the integrity of this organelle. In this case, AC would have a hormetic effect on MMP: namely, MMP would have a biphasic response to $\mathrm{AC}$, which would be characterized by a response effect consisting of an increase at low doses, and a decrease at the high ones. Finally, the lack of a concomitant increase in $\mathrm{H}_{2} \mathrm{O}_{2}$ levels might be explained by the normal efflux of this molecule, since rather than targeting GLPs, which present oxygen peroxide permeability, AC inhibit orthodox AQPs only.

\section{Conclusion}

In conclusion, AQP-inhibition effects are highly reliant upon the specificity of the inhibitor and its ability to affect other sperm proteins. In this work, PDO has been found to improve post-thaw sperm survival at the expense of an increase in the percentages of viable spermatozoa with high ROS levels, which suggests its potential role as a CPA. Further research is, however, required to confirm this hypothesis. Based on the observed effects after AC supplementation, which mainly targets orthodox AQPs, it seems that orthodox AQPs are not involved in the response to osmolality variations during freeze-thawing of boar sperm, since the observed changes compared to the control seem to result from collateral effects of this inhibitor upon the sperm cell. Finally, the dramatic impairment observed in post-thaw sperm quality parameters when PHL was added to the cryopreservation media supports the crucial role of GLPs - and not of orthodox AQPs - during boar sperm cryopreservation.

\section{Abbreviations \\ AC: Acetazolamide; ALH: Amplitude of lateral head displacement; AQP: Aquaporin; BCF: Beat cross frequency; BTS: Beltsville Thawing Solution; CA: Carbonic anhydrase; CAMP: cyclic AMP; CASA: Computer- assisted sperm analysis; CPA: Cryoprotective agent; $\mathrm{DCF}^{+}$: $2^{\prime}, 7^{\prime}-$ dichlorofluorescein; $E^{+}$: Ethidium; EV: Electronic volume; FS: Forward scatter; GLP: Aquaglyceroporins; $\mathrm{H}_{2}$ DCFDA: 2',7'-dichlorodihydrofluorescein diacetate; $\mathrm{H}_{2} \mathrm{O}_{2}$ : Hydrogen peroxide; HE: Hydroethidine; ISAC: International Society for Advancement of Cytometry; JC-1: 5,5',6,6'-tetrachloro-1,1',3,3'tetraethyl- benzimidazolylcarbocyanine iodide; JC-1 agg: JC-1 aggregates; JC-1 mon: JC-1 monomers; LEY: $\beta$-Lactose -Egg yolk -Glycerol; LEYGO: $\beta$-Lactose -Egg yolk -Glycerol -Orvus Es Paste; LIN: Linearity; M540: Merocyanine 540; MMP: Mitochondrial membrane potential; $\mathrm{O}_{2}^{-} \cdot$ Superoxide; PDO: 1,3- propanediol; PfAQP: Plasmodium falciparum aquaporin; PHL: Phloretin; PI: Propidium iodide; PKA: CAMP dependent protein kinase; PKC: Protein kinase C; PMOT: Progressive motility; ROS: Reactive oxygen species; SEM: Standard error of the mean; SLC2A2: Solute carrier family 2, facilitated glucose transporter member 2; SS: Side scatter; STR: Straightness; TMOT: Total motility; VAP: Average path velocity; VCL: Curvilinear velocity; VSL: Straight line velocity; WOB: Motility parameter wobble}

\section{Acknowledgements}

The authors acknowledge the technical support received from Estela Garcia (University of Girona, Spain).

\section{Authors' contributions}

ADB performed the experiments, undertook flow cytometry and statistical analyses and wrote the draft. ML and LFB contributed to performing CASA and flow cytometry evaluations. IB, SR, YM, SB and BFF contributed to the critical revision of the Manuscript. MY conceived the study and help conduct data analysis. All authors read and approved the final manuscript.

\section{Funding}

The authors acknowledge the support from the European Commission (H2020-MSCA-IF-79212), the Ministry of Science, Innovation and Universities, Spain (Grants: RYC-2014-15581, AGL2016-81890-REDT, AGL2017-88329-R and FJCl-2017-31689), and the Regional Government of Catalonia, Spain (2017SGR-1229).

\section{Availability of data and materials}

The datasets and/or analyzed during the current study are available from the corresponding author on reasonable request. 


\section{Ethics approval and consent to participate}

Not applicable.

\section{Consent for publication}

Not applicable.

\section{Competing interests}

The authors declare that the research was conducted in the absence of any commercial or financial relationships that could be construed as a potential conflict of interest.

\section{Received: 17 April 2019 Accepted: 8 August 2019}

Published online: 14 October 2019

\section{References}

1. Watson H. Biological membranes. Essays Biochem. 2015;59:43-70.

2. Yeste M, Morató R, Rodríguez-Gil JE, Bonet S, Prieto-Martínez N. Aquaporins in the male reproductive tract and sperm: functional implications and cryobiology. Reprod Domest Anim. 2017;52:12-27.

3. Prieto-Martínez N, Vilagran I, Morató R, Rodríguez-Gil JE, Yeste M, Bonet S. Aquaporins 7 and 11 in boar spermatozoa: detection, localisation and relationship with sperm quality. Reprod Fertil Dev. 2014;28:663-72.

4. Prieto-Martínez N, Morató R, Vilagran I, Rodríguez-Gil JE, Bonet S, Yeste M. Aquaporins in boar spermatozoa. Part II: detection and localisation of aquaglyceroporin 3. Reprod Fertil Dev. 2015;29:703-11.

5. Chen Q, Peng H, Lei L, Zhang Y, Kuang H, Cao Y, et al. Aquaporin 3 is a sperm water channel essential for postcopulatory sperm osmoadaptation and migration. Cell Res. 2011;21:922-33.

6. Yeung $\mathrm{CH}$, Callies $\mathrm{C}$, Rojek A, Nielsen S, Cooper TG. Aquaporin isoforms involved in physiological volume regulation of murine spermatozoa. Biol Reprod. 2009;80:350-7

7. Yeung $\mathrm{CH}$, Cooper TG. Aquaporin AQP11 in the testis: molecular identity and association with the processing of residual cytoplasm of elongated spermatids. Reproduction. 2010;139:209-16.

8. Yeung $\mathrm{CH}$, Callies C, Tüttelmann F, Kliesch S, Cooper TG. Aquaporins in the human testis and spermatozoa - identification, involvement in sperm volume regulation and clinical relevance. Int J Androl. 2010:33:629-41.

9. Laforenza U, Pellavio G, Marchetti A, Omes C, Todaro F, Gastaldi G Aquaporin-mediated water and hydrogen peroxide transport is involved in Normal human spermatozoa functioning. Int J Mol Sci. 2017;18:E66.

10. Prieto-Martínez N, Morató R, Muiño R, Hidalgo CO, Rodríguez-Gil JE, Bonet S, et al. Aquaglyceroporins 3 and 7 in bull spermatozoa: identification, localisation and their relationship with sperm cryotolerance. Reprod Fertil Dev. 2016;29:1249-59.

11. Morató R, Prieto-Martínez N, Muiño R, Hidalgo CO, Rodríguez-Gil JE, Bonet S, et al. Aquaporin 11 is related to cryotolerance and fertilising ability of frozen-thawed bull spermatozoa. Reprod Fertil Dev. 2018;30:1099-108.

12. Bonilla-Correal S, Noto F, Garcia-Bonavila E, Rodríguez-Gil JE, Yeste M, Miró J. First evidence for the presence of aquaporins in stallion sperm. Reprod Domest Anim. 2017:52:61-4

13. Vicente-Carrillo A, Ekwall H, Álvarez-Rodríguez M, Rodríguez-Martínez H. Membrane stress during thawing elicits redistribution of aquaporin 7 but not of aquaporin 9 in boar spermatozoa. Reprod Domest Anim. 2016;51:665-79.

14. Huang HF, He RH, Sun CC, Zhang Y, Meng QX, Ma YY. Function of aquaporins in female and male reproductive systems. Hum Reprod Update. 2006;12:785-95

15. Yeste M. Sperm cryopreservation update: Cryodamage, markers, and factors affecting the sperm freezability in pigs. Theriogenology. 2016;85:47-64.

16. Bonet S, Casas I, Holt W. Boar reproduction: fundamentals and new biotechnological trends. 1st ed. London: Springer; 2013.

17. Yeste M. Recent advances in boar sperm cryopreservation: state of the art and current perspectives. Reprod Domest Anim. 2015;50:71-9.

18. Yeste M, Rodríguez-Gil JE, Bonet S. Artificial insemination with frozen-thawed boar sperm. Mol Reprod Dev. 2017;84:802-13.

19. Prieto-Martínez N, Vilagran I, Morató R, Rivera del Álamo MM, Rodríguez-Gil JE, Bonet S, et al. Relationship of aquaporins 3 (AQP3), 7 (AQP7), and 11 (AQP11) with boar sperm resilience to withstand freeze-thawing procedures. Andrology. 2017;5:1153-64.

20. Yu L, Villarreal OD, Chen LL, Chen LY. 1,3-Propanediol binds inside the water-conducting pore of aquaporin 4: does this efficacious inhibitor have sufficient potency? J Syst Integr Neurosci. 2016;2:91-8.
21. Yu L, Rodriguez RA, Chen LL, Chen LY, Perry G, McHardy SF, et al. 1,3Propanediol binds deep inside the channel to inhibit water permeation through Aquaporins. Protein Sci. 2016;25:433-41.

22. Gao J, Wang X, Chang Y, Zhang J, Song Q, Yu H, et al. Acetazolamide inhibits osmotic water permeability by interaction with aquaporin-1. Anal Biochem. 2006;350:165-70.

23. Tanimura Y, Hiroaki Y, Fujiyoshi Y. Acetazolamide reversibly inhibits water conduction by aquaporin-4. J Struct Biol. 2009;166:16-21.

24. Tsukaguchi H, Shayakul C, Berger UV, Mackenzie B, Devidas S, Guggino WB, et al. Molecular characterization of a broad selectivity neutral Solute Channel. J Biol Chem. 1998;273:24737-43.

25. Cheung $\mathrm{KH}$, Leung $\mathrm{CT}$, Leung GPH, Wong PYD. Synergistic effects of cystic fibrosis transmembrane conductance regulator and Aquaporin-9 in the rat epididymis. Biol Reprod. 2003;68:1505-10.

26. Casas I, Sancho S, Briz M, Pinart E, Bussalleu E, Yeste M, et al. Freezability prediction of boar ejaculates assessed by functional sperm parameters and sperm proteins. Theriogenology. 2009:72:930-48.

27. Pursel VG, Johnson LA. Freezing of boar spermatozoa: fertilizing capacity with concentrated semen and a new thawing procedure. J Anim Sci. 1975; 40:99-102.

28. Garner DL, Johnson LA. Viability assessment of mammalian sperm using SYBR-14 and propidium iodide. Biol Reprod. 1995;53:276-84.

29. Rathi R, Colenbrander B, Bevers MM, Gadella BM. Evaluation of in vitro capacitation of stallion spermatozoa. Biol Reprod. 2001;65:462-70.

30. Yeste M, Estrada E, Rivera Del Álamo MM, Bonet S, Rigau T, Rodríguez-Gil JE. The increase in phosphorylation levels of serine residues of protein HSP70 during holding time at $17^{\circ} \mathrm{C}$ is concomitant with a higher cryotolerance of boar spermatozoa. PLoS One. 2014;9:e90887.

31. Ortega-Ferrusola C, Sotillo-Galán Y, Varela-Fernández E, Gallardo-Bolaños JM, Muriel A, González-Fernández L, et al. Detection of 'apoptosis-like' changes during the cryopreservation process in equine sperm. J Androl. 2008;29:213-21.

32. Guthrie HD, Welch GR. Determination of intracellular reactive oxygen species and high mitochondrial membrane potential in Percoll-treated viable boar sperm using fluorescence-activated flow cytometry. J Anim Sci. 2006;84:2089-100

33. Rezk BM, Haenen GRMM, van der Vijgh WJF, Bast A. The antioxidant activity of phloretin: the disclosure of a new antioxidant pharmacophore in flavonoids. Biochem Biophys Res Commun. 2002;295:9-13.

34. Barreca D, Currò M, Bellocco E, Ficarra S, Laganà G, Tellone E, et al. Neuroprotective effects of phloretin and its glycosylated derivative on rotenone-induced toxicity in human SH-SY5Y neuronal-like cells. BioFactors. 2017:43:549-57.

35. Przybylo M, Procek J, Hof M, Langner M. The alteration of lipid bilayer dynamics by phloretin and 6-ketocholestanol. Chem Phys Lipids. 2014;178:38-44.

36. Cooper TG, Barfield JP, Yeung $\mathrm{CH}$. The tonicity of murine epididymal spermatozoa and their permeability towards common cryoprotectants and epididymal osmolytes. Reproduction. 2008;135:625-33.

37. Widiasih D, Yeung $\mathrm{CH}$, Junaidi A, Cooper TG. Multistep and single-step treatment of human spermatozoa with cryoprotectants. Fertil Steril. 2009;92:382-9.

38. Lopes CA, Alves AM, Jewgenow K, Báo SN, de Figueiredo JR. Cryopreservation of canine ovarian cortex using DMSO or 1,3-propanediol. Theriogenology. 2016:86:1165-74

39. Pogozhykh D, Prokopyuk V, Pogozhykh O, Mueller T, Prokopyuk O. Influence of factors of cryopreservation and hypothermic storage on survival and functional parameters of multipotent stromal cells of placental origin. PLoS One. 2015;10:e0139834.

40. Aitken RJ. Reactive oxygen species as mediators of sperm capacitation and pathological damage. Mol Reprod Dev. 2017:84:1039-52

41. Miller EW, Dickinson BC, Chang CJ. Aquaporin-3 mediates hydrogen peroxide uptake to regulate downstream intracellular signaling. Proc Natl Acad Sci. 2010:107:15681-6.

42. Watanabe S, Moniaga CS, Nielsen S, Hara-Chikuma M. Aquaporin-9 facilitates membrane transport of hydrogen peroxide in mammalian cells. Biochem Biophys Res Commun. 2016:471:191-7.

43. Pohl P, Rokitskaya TI, Pohl EE, Saparov SM. Permeation of phloretin across bilayer lipid membranes monitored by dipole potential and microelectrode measurements. Biochim Biophys Acta. 1997:1323:163-72.

44. Wacker SJ, Aponte-Santamaría C, Kjellbom P, Nielsen S, De Groot BL, Rützler $M$. The identification of novel, high affinity AQP9 inhibitors in an intracellular binding site. Mol Membr Biol. 2013;30:246-60. 
45. Fan H, Morishima S, Kida H, Okada Y. Phloretin differentially inhibits volumesensitive and cyclic AMP-activated, but not ca-activated, cl-channels. Br J Pharmacol. 2001;133:1096-106.

46. Bucci D, Isani G, Spinaci M, Tamanini C, Mari G, Zambelli D, et al. Comparative Immunolocalization of GLUTS 1, 2, 3 and 5 in boar, stallion and dog spermatozoa. Reprod Domest Anim. 2010;45:315-22.

47. Van Berkel MA, Pharm D. Evaluating off-label uses of acetazolamide. Am J Health Syst Pharm. 2018;75:524-31.

48. Jakobsen E, Lange SC, Andersen JV, Desler C, Kihl HF, Hohnholt MC, et al. The inhibitors of soluble adenylate cyclase 2-OHE, KH7, and bithionol compromise mitochondrial ATP production by distinct mechanisms. Biochem Pharmacol. 2018;155:92-101.

Ready to submit your research? Choose BMC and benefit from:

- fast, convenient online submission

- thorough peer review by experienced researchers in your field

- rapid publication on acceptance

- support for research data, including large and complex data types

- gold Open Access which fosters wider collaboration and increased citations

- maximum visibility for your research: over $100 \mathrm{M}$ website views per year

At BMC, research is always in progress.

Learn more biomedcentral.com/submissions 